\title{
HBV suppresses thapsigargin-induced apoptosis via inhibiting CHOP expression in hepatocellular carcinoma cells
}

\author{
DANQI ZHAO ${ }^{*}$, YAN LIU* ${ }^{*}$ XING LIU, TAO LI, ZHENHUI XIN, XILIN ZHU, XIAOPAN WU and YING LIU \\ National Laboratory of Medical Molecular Biology, Institute of Basic Medical Sciences, \\ Chinese Academy of Medical Sciences; School of Basic Medicine, Peking Union Medical College, Beijing 100005, P.R. China
}

Received April 18, 2016; Accepted July 21, 2017

DOI: $10.3892 / \mathrm{ol} .2017 .6666$

\begin{abstract}
Hepatocellular carcinoma (HCC) accounts for a proportion of cancer-associated mortalities worldwide. Hepatitis B virus (HBV) infection is a major cause of $\mathrm{HCC}$ in China. Thapsigargin (TG) is a potential antitumor prodrug, eliciting endoplasmic reticulum (ER) stress via the inhibition of the ER calcium pump, effectively inducing apoptosis. The present study therefore examined the role of HBV in TG-induced apoptosis using two HCC cell lines, HBV positive HepG2.2.15 and HBV negative HepG2. When these two cell lines were treated with TG, HepG2.2.15 was less susceptible to apoptosis than HepG2. This phenomenon was confirmed by an MTT assay and Annexin V-FITC/propidium iodide staining. Reverse transcription quantitative polymerase chain reaction and western blotting were used to detect the expression levels of genes in the ER stress pathway subsequent to treatment with TG. Notably, the mRNA and protein levels of the apoptosis factor DNA damage inducible transcript 3 (CHOP) increased significantly in the HepG 2 cells compared with the HepG2.2.15 cells. Additionally, the HepG2.2.15 cells treated with interferon- $\alpha$ exhibited higher levels of CHOP
\end{abstract}

Correspondence to: Professor Xiaopan Wu or Professor Ying Liu, National Laboratory of Medical Molecular Biology, Institute of Basic Medical Sciences, Chinese Academy of Medical Sciences; School of Basic Medicine, Peking Union Medical College, 5 Dongdan 3 Tiao, Dongcheng, Beijing 100005, P.R. China

E-mail:wuxiaopanpumc@163.com

E-mail: liuyingpumc2016@sina.com

*Contributed equally

Abbreviations: $\mathrm{HBV}$, hepatitis B virus; HCC, hepatocellular carcinoma; ER, endoplasmic reticulum; UPR, unfolded protein response; TG, thapsigargin; PERK, protein kinase-like endoplasmic reticulum kinase; IRE1, inositol-requiring enzyme 1; ATF4, activating transcription factor 4; ATF6, activating transcription factor 6; CHOP, DNA damage inducible transcript 3; GADD34, protein phosphatase 1 regulatory subunit $15 \mathrm{~A}$

Key words: HBV, thapsigargin, apoptosis, CHOP, endoplasmic reticulum stress compared with the untreated cells. The overexpression or knockdown of CHOP microRNA in HepG2.2.15 or HepG2 cells may reduce the difference in apoptosis status between the two cell lines. These results suggest that HBV may inhibit the apoptosis induced by ER stress. These findings may be useful in the development of selective therapies for patients with HBV-positive tumors.

\section{Introduction}

Thapsigargin (TG) is a natural product isolated from the seeds of Thapsia garganica $L$, which binds tightly to and inhibits the function of the transmembrane portion of the sarcoplasmic/endoplasmic reticulum calcium adenosine triphosphatase pump, inducing apoptosis $(1,2)$. TG initiates endoplasmic reticulum (ER) stress via the unfolded protein response (UPR), which is initiated by 3 ER transmembrane proteins termed protein kinase-like endoplasmic reticulum kinase (PERK), inositol-requiring enzyme 1 (IRE1) and activating transcription factor (ATF) $6(3,4)$. An abnormality of this network affects the progression of various types of cancer, such as breast cancer, pancreatic adenocarcinoma and melanoma $(3,5)$.

A TG prodrug that is activated in the vasculature of solid tumors by tumor endothelial cells has been developed (6). It is highly selective to tumor endothelial cells, and the drug toxicity level is expected to be low. This TG prodrug is useful in the majority of types of solid tumors with prostate-specific membrane antigen expression, including hepatocellular carcinoma (HCC) (7). Several factors contribute to HCC, such as chronic hepatitis B infection, excessive alcohol consumption and other chronic hepatic damage (8). These factors may cause oxidative stress, inflammation and mutation, which transform hepatic cells into HCC cells by inducing ER stress (9-11). In China, numerous patients with HCC are hepatitis B virus (HBV) carriers (12). HBV infection causes massive viral replication and produces a large number of viral proteins in a short period of time, which results in the disturbance of ER homeostasis and therefore protein misfolding. The accumulation of unfolded or misfolded proteins leads to ER stress, followed by UPR (13). However, only a limited number of studies have investigated the effect of HBV on UPR gene expression and apoptosis $(14,15)$. The present study used two cell lines, HepG2 and HepG2.2.15, 
which are HBV negative and positive, respectively, to study whether HBV affects apoptosis during ER stress induced by TG.

\section{Materials and methods}

Cell culture, reagents and antibodies. The HepG2 cells were purchased from the Chinese Academy of Medical Sciences Cell Culture Center (Beijing, China) and the HepG2.2.15 cells were purchased from China Center for Type Culture Collection (Wuhan, China). The cells were cultured in Dulbecco's modified Eagle's medium (Gibco; Thermo Fisher Scientific Inc., Waltham, MA, USA) with $10 \%$ fetal bovine serum (FBS; Gibco; Thermo Fisher Scientific, Inc.). The cell lines were maintained in an atmosphere with $5 \% \mathrm{CO}_{2}$ and saturated humidity at $37^{\circ} \mathrm{C}$. Images of the cells were captured using a CKX41 microscope (Olympus Corporation, Tokyo, Japan) at a magnification of $\mathrm{x} 100$ using the TopTek ToupView version 3.7 (OPTEC, Chongqing, China). TG was obtained from Sigma-Aldrich; Merck Millipore (Darmstadt, Germany; cat. no., 586005-1MG), dissolved in dimethylsulfoxide (DMSO; Amresco, LLC., Solon, OH, USA) and used at a dilution of $500 \mathrm{nM}$.

A total of 200 units/well recombinant human interferon (IFN) $\alpha-2 \mathrm{~A}$ (Fangcheng BioTech Co Ltd; Beijing, China; cat. no., CYT-204) was added $6 \mathrm{~h}$ prior to TG treatment. MTT was purchased from Sigma-Aldrich; Merck Millipore (cat no. M2128) and was used at a concentration of $5 \mathrm{mg} / \mathrm{ml}$. PBS was purchased from Tianjin TBD Haoyang BioTech Co Ltd (Tianjin, China; cat. no., PB2004Y). A eukaryotic DNA damage inducible transcript 3 (CHOP) expression plasmid encoding the full-length $\mathrm{CHOP}$ protein was purchased from Tianyi Huiyuan Biotechnology Co., (Wuhan, China). Small interfering RNA (siRNA) for the CHOP protein was purchased from Guangzhou RiboBio Co., Ltd., (Guangzhou, China). Lipofectamine 2000 was purchased from Invitrogen; Thermo Fisher Scientific, Inc., (cat. no., 1609073). The Ultrapure RNA (cat. no., CW0581), Protein Extraction (cat. no., cw0889), SYBR Green polymerase chain reaction (PCR; cat. no., CW2601), Annexin V-FITC/PI Apoptosis Detection (cat. no., CW2574) and Cell Cycle and Apoptosis Detection (cat. no., CW2575) kits were purchased from CWBio (Beijing, China). The ReverTra Ace reverse transcription quantitative (RT-q)PCR Master Mix was purchased from Toyobo Co., Ltd. (Osaka, Japan; cat. no., FSQ-201). Antibodies against CHOP (polyclonal rabbit, cat. no., sc-575) and glyceraldehyde-3-phosphate dehydrogenase (GAPDH; polyclonal rabbit, cat. no., CW0101) were purchased from Santa Cruz Biotechnology, Inc., (Santa Cruz; Dallas, TX, USA) and CWBIO (Beijing, China), respectively. Horseradish peroxidase conjugated goat anti rabbit IgG was purchased from CWBio (cat. no., CW103A).

MTT assay. The HepG2 or HepG2.2.15 cells were seeded at a density of $5 \times 10^{3}$ cells/well in 96-well plates. The cells were treated with TG for 24, 48, 72 and $96 \mathrm{~h}$ and cells treated with PBS were used as controls. Subsequent to the end of each time point, the cells were incubated with $10 \mu \mathrm{l}$ MTT for $4 \mathrm{~h}$ at $37^{\circ} \mathrm{C}$ in the dark. The supernatant was removed and $100 \mu$ l DMSO was used for dissolution. A Synergy H1 microplate reader
(BioTek; Winooski, VT, USA) was used to measure absorbance at $490 \mathrm{~nm}$. All experiments were performed in triplicate and repeated 3 times.

Flow cytometry assay. A flow cytometry assay was used to investigate the level of apoptosis and the cell cycle of the HepG2 and HepG2.2.15 cells. The HepG2 cells were divided into 2 groups. The cells in the first group were treated with TG for 24, 48, 72 and $96 \mathrm{~h}$. The untreated cells in the second group were used as controls. The HepG2.2.15 cells were divided into 3 groups. The cells in the first group were treated with IFN $\alpha-2 \mathrm{~A}$ for $6 \mathrm{~h}$ and incubated with TG for $24,48,72$ and $96 \mathrm{~h}$ at $37^{\circ} \mathrm{C}$. The cells in the second group were treated with TG for 24, 48, 72 and $96 \mathrm{~h}$ directly. The untreated cells in the third group were used as controls. For the apoptosis assays, the cells were incubated with propidium iodide (PI) and Annexin V-FITC at $24^{\circ} \mathrm{C}$ for $15 \mathrm{~min}$ according to the protocol of the Annexin V-FITC/PI Apoptosis Detection kit. For the cell cycle assays, the collected cells were treated according to the protocol provided with the Cell Cycle and Apoptosis Detection kit. Apoptosis and cell cycle were quantified and analyzed using the Accuri C6 flow cytometer (BD Biosciences, Franklin Lakes, NJ, USA).

Quantitative analysis of gene expression. The HepG2 cells were seeded in 24-well plates at a density of $2 \times 10^{5} /$ well for a RT qPCR analysis. Each well of HepG2 or HepG2.2.15 cells was incubated with $500 \mathrm{nM}$ TG. The HepG2 or HepG2.2.15 cells were treated with TG for $24,48,72$ and $96 \mathrm{~h}$ at $37^{\circ} \mathrm{C}$. Untreated cells were used as controls. All treatments were performed in triplicate and repeated 3 times. The cells were collected at each time point and total RNA extraction was performed using the Ultrapure RNA kit according to the protocol of the manufacturer. Complementary DNA was synthesized in a $10 \mu \mathrm{l}$ reaction volume using ReverTra Ace qPCR RT Master Mix following the protocol of the manufacturer. The mRNA expression levels of ATF6, ATF4, CHOP and protein phosphatase 1 regulatory subunit 15A (GADD34) were measured by a SYBR Green relative quantitative analysis using the Bio-Rad iQ5 Real-Time PCR Detection System (Bio-Rad Laboratories, Inc., Hercules, CA, USA). GAPDH was used as an internal control. The primers and probes used for the qPCR are listed in Table I. The RT qPCR conditions were as follows: $95^{\circ} \mathrm{C}$ for $10 \mathrm{~min}$, followed by 40 cycles of $92^{\circ} \mathrm{C}$ for $15 \mathrm{sec}$ and $58^{\circ} \mathrm{C}$ for $1 \mathrm{~min}$. The relative expression levels of genes were calculated by the $2^{-\Delta \Delta \mathrm{Ct}}$ method (16) and normalized by the level of the internal control. X-box protein 1 (XBP1) microRNA (mRNA) splicing was detected by RT qPCR as described previously (17). Theoretically, a 289 bp amplicon is generated from unspliced XBP1, XBP1 ${ }^{u}$, while a $263 \mathrm{bp}$ amplicon is generated from spliced XBP1, $\mathrm{XBP}^{\mathrm{s}}(17)$.

Overexpression or knockdown of CHOP. A total of $5 \times 10^{3}$ HepG2 or HepG2.2.15 cells were seeded in each well of a 96-well plate. The cells were transfected with $0.1 \mu \mathrm{g}$ of CHOP expression plasmids or $50 \mathrm{nM}$ siRNA with $0.2 \mu \mathrm{l}$ Lipofectamine 2000. The cells transfected with only Lipofectamine 2000 were set as controls. A total of $6 \mathrm{~h}$ subsequent to transfection, $500 \mathrm{nM}$ TG was added to the 
Table I. Primers used in the mRNA quantification.

Primers and probes $\quad$ Sequence (5'-3')

ATF6

Forward primer of PCR CCCAAGACTCAAACAAACTC

Reverse primer of PCR GTGATTAGGGAGCTGTGTGA

ATF4

Forward primer of PCR

GTGTGCGTTTTCCCTCCTC

Reverse primer of PCR

TGTCGGTTACAGCAACGCT

XBP1

Forward primer of PCR

TTACGAGAGAAAACTCAT

GGC

Reverse primer of PCR

GGGTCCAAGTTGTCCAGAA TGC

\section{CHOP}

Forward primer of PCR

Reverse primer of PCR

GADD34

Forward primer of PCR

Reverse primer of PCR

GAPDH

Forward primer of PCR

Reverse primer of PCR

\section{TCTAAGGCACTGAGCGTATC} CAGTCTGGAAAAGCACATCT

\section{CCAGAAACCCCTACTCATG CAGGAAATGGACAGTGACC}

TCTGACTTCAACAGCGACAC CAAATTCGTTGTCATACCAG

culture medium. The cells were then collected at 24, 48, 72 and $96 \mathrm{~h}$ for the MTT assay.

Western blotting. The proteins were extracted using the Mammalian Protein Extraction kit, separated by $10 \%$ SDS-PAGE, and transferred to a polyvinylidene fluoride membrane (cat. no., ISEQ00010; EMD Millipore, Billerica, MA, USA). Subsequent to blocking with 5\% non-fat powdered milk, the membranes were incubated with antibodies against CHOP (dilution, 1:1,000) and GAPDH (dilution, 1:10,000) at $4{ }^{\circ} \mathrm{C}$ overnight. The membranes were washed with TBS containing $0.1 \%$ Tween 20 and stained with the 1:10,000 IgG at $37^{\circ} \mathrm{C}$ for $1 \mathrm{~h}$. Micrographs were captured using the Tanon 5200 Multi (Tanon Science and Technology Co., Ltd., Shanghai, China).

Statistical analysis. Spliced XBP1 mRNA as a percentage of the total XBP1 mRNA was estimated using ImageJ software 1.4r (National Institutes of Health, Bethesda, MD, USA). Statistical analyses were performed using SPSS v.15.0 (SPSS, Inc., Chicago, IL, USA) and all data were analyzed by a one-way analysis of variance from three independent experiments. P-values were determined using unpaired Student's t-test. $\mathrm{P}<0.05$ was considered to indicate a statistically significant difference.

\section{Results}

HBV-positive HepG2.2.15 cells are less susceptible to apoptosis. HBV-positive HepG2.2.15 and HBV negative HepG2 cells were seeded in 24 -well plates. Subsequent to

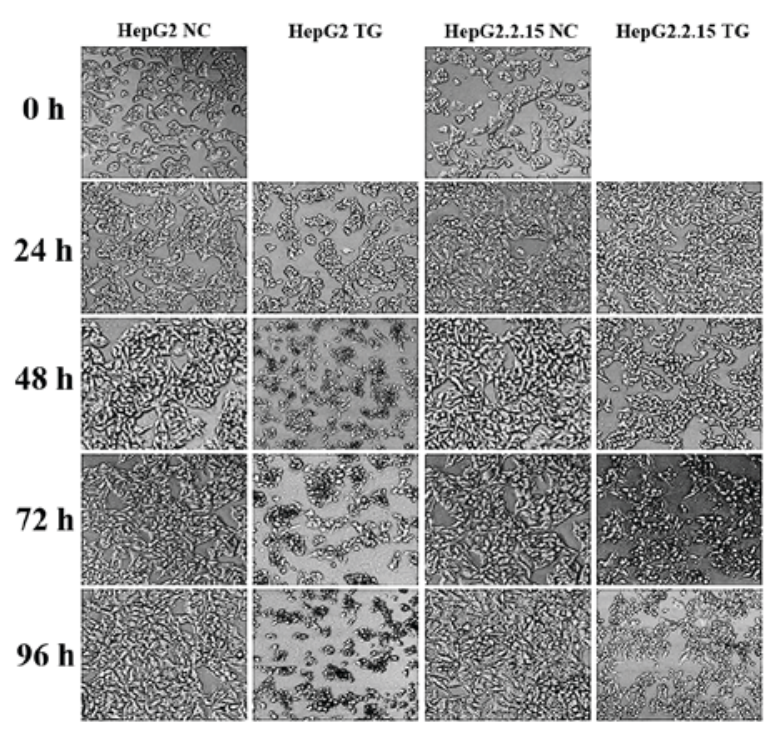

Figure 1. Morphology of HepG2 and HepG2.2.15 cells viewed under a microscope. Magnification x100. NC, negative control; TG, thapsigargin.

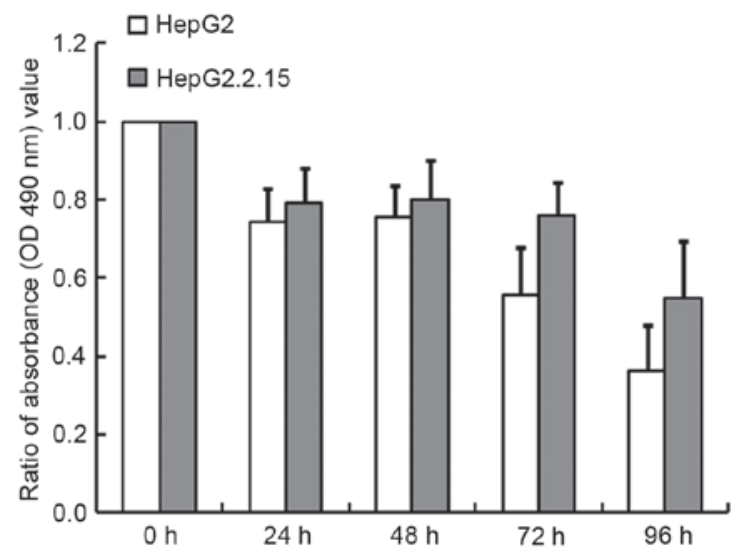

Figure 2. MTT assay results for the HepG2 and HepG2.2.15 cells treated with thapsigargin (TG) or PBS. Final abundance was calculated as the ratio of absorbance at $490 \mathrm{~nm}$ for the cells treated with TG relative to the cells treated with PBS at each time point. Data are presented as mean \pm standard deviation of three independent experiments. OD, optical density.

1 day, $500 \mathrm{nM}$ TG or PBS was added to the experimental or control groups respectively. As demonstrated in Fig. 1, the HepG2 and HepG2.2.15 cells treated with PBS exhibited normal morphologies, with clear cell membrane boundaries and homogeneous cell cytoplasm densities, whilst the two cell types showed apoptosis when treated with TG. Notably, the HepG2 cells underwent more apoptosis than HepG2.2.15 cells, by morphological observation. Accordingly, it was hypothesized that HBV may alleviate the apoptosis induced by TG.

$H B V$ may repress apoptosis induced by ER stress via the CHOP pathway. To determine whether HBV affects cell survival and apoptosis, the HepG2.2.15 and HepG2 cells were treated with $500 \mathrm{nM}$ TG or PBS for 24, 48, 72 and $96 \mathrm{~h}$ and cell proliferation was detected using the MTT assay. As illustrated in Fig. 2, TG inhibited cell growth in a time-dependent manner, especially in the HepG2 cells, for which $64 \%$ cell growth was inhibited at $96 \mathrm{~h}$ compared with $45 \%$ in the HepG2.2.15 cells 

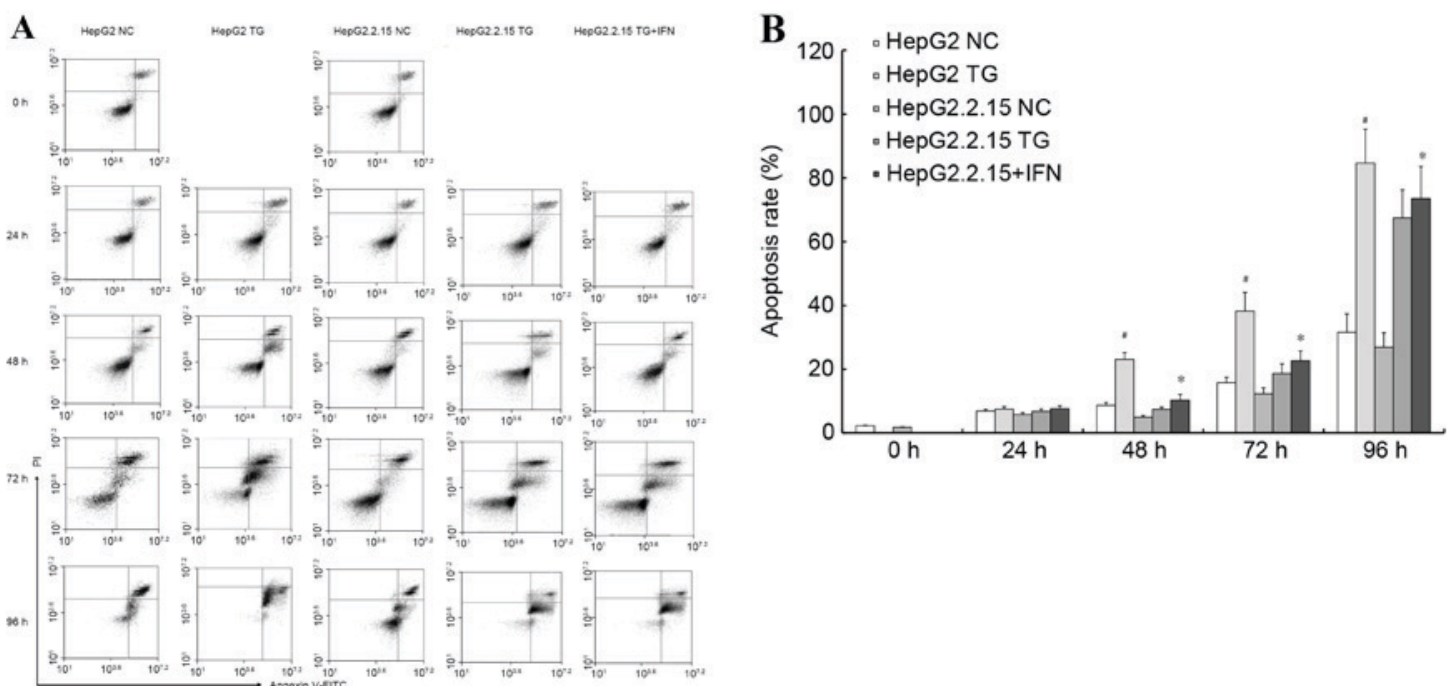

Figure 3. (A) Apoptosis of the HepG2 and HepG2.2.15 cells detected by flow cytometry assay. (B) TG increased the apoptotic populations of HepG2 cells more significantly compared with HepG2.2.15 cells. The data are presented as the mean \pm standard deviation from three independent experiments, and P-values were determined using an unpaired Student's t-test. ${ }^{\#} \mathrm{HepG} 2$ cells treated with TG vs. HepG2.2.15 cells treated with TG $(\mathrm{P}<0.01) .{ }^{*} \mathrm{HepG} 2.2 .15$ cells treated with IFNa-2A and TG vs. HepG2.2.15 cells treated with TG $(\mathrm{P}<0.05)$. NC, negative control; TG, thapsigargin; IFN, interferon.
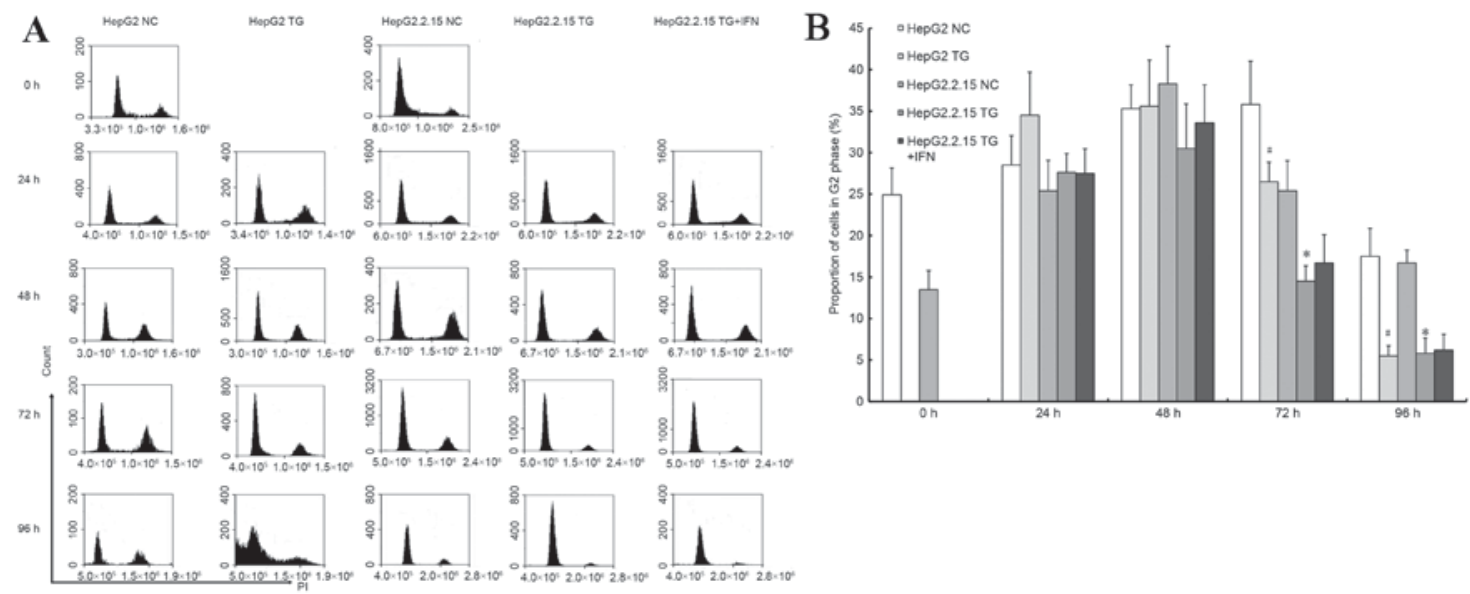

Figure 4. (A) Cell cycles of the HepG2 and HepG2.2.15 cells detected by flow cytometry assay. (B) TG reduced the proportion of cells in G2 phase. Data are presented as the mean \pm standard deviation. ${ }^{~ H e p G 2 ~ c e l l s ~ v s . ~ H e p G 2 ~ c e l l s ~ t r e a t e d ~ w i t h ~ T G ~}(\mathrm{P}<0.05)$. "HepG2.15 cells vs. HepG2.2.15 cells treated with TG $(\mathrm{P}<0.05)$. PI, propidium iodide; $\mathrm{NC}$, negative control; TG, thapsigargin; IFN, interferon.

$(\mathrm{P}<0.001)$. Similarly, at 24, 48 and $72 \mathrm{~h}$, greater inhibition was observed for the HepG2 cells than for HepG2.2.15 cells. These results indicated that $\mathrm{HBV}$ may alleviate the induction of apoptosis by TG.

Using annexin V/PI double staining, the effect of TG on apoptosis in the cells was then examined. Using flow cytometry, and demonstrated in Fig. 3A, it was revealed that TG increased the apoptotic populations of HepG2 cells more significantly $(\mathrm{P}<0.01)$ compared with the HepG2.2.15 cells, as illustrated in Fig. 3B. In addition, to confirm the role of $\mathrm{HBV}$, the HepG2.2.15 cells were treated with IFN $\alpha-2 \mathrm{~A}$, an anti-HBV drug, and the aforementioned experiments were repeated. When the HepG2.2.15 cells were treated with IFN $\alpha-2 \mathrm{~A}$ and TG the HBV load decreased and the apoptosis rate increased compared with the HepG2.2.15 cells treated with TG $(\mathrm{P}<0.05)$, as demonstrated in Fig. 3B. PI staining was used to detect the cell cycle, as demonstrated in Fig. 4A.
TG reduced the proportion of $\mathrm{G} 2$ phase cells $(\mathrm{P}<0.05)$, as illustrated in Fig. 4B. In the HepG2 cells, there were significant sub-apoptosis peaks at $96 \mathrm{~h}$, which indicated that HBV may induce apoptosis to a lesser degree. Based on the cell cycle experiment, the HepG2.2.15 cells treated with IFN $\alpha-2 \mathrm{~A}$ and TG exhibited sub-apoptosis peaks at $96 \mathrm{~h}$, similar to the HepG2 cells, as illustrated in Fig. 4B. However, no difference was observed in the results of the MTT experiment with respect to IFN $\alpha-2 \mathrm{~A}$ treatment: The ratio of the HepG2.2.15 cells treated with TG and IFN $\alpha-2 \mathrm{~A}$ to the cells treated with $\mathrm{TG}$ alone at each time point was between 0.99 and 1.03. These results demonstrated that a reduced HBV load in the HepG2.2.15 cells may increase apoptosis, or HBV may inhibit the apoptosis induced by TG.

To explore the mechanism underlying the associations between HBV, TG and apoptosis, the mRNA levels of 5 genes in 3 UPR pathways: The ATF6 gene in the ATF6 pathway; the 
$\mathbf{A}$

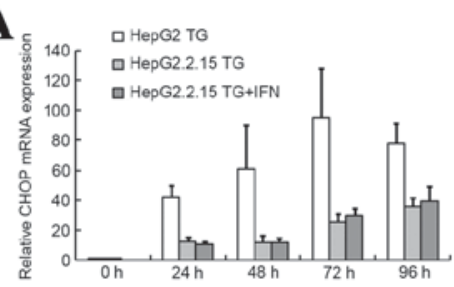

口. HepG2 TG

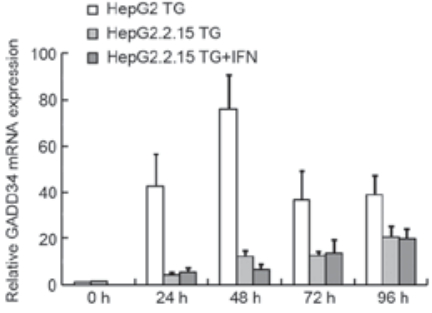

HepG2 TG

口 HepG2.2.15 TC

口 HePG2.2.15 TG+IFN

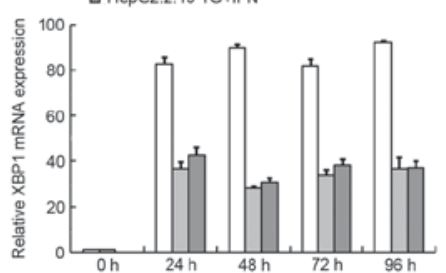

B
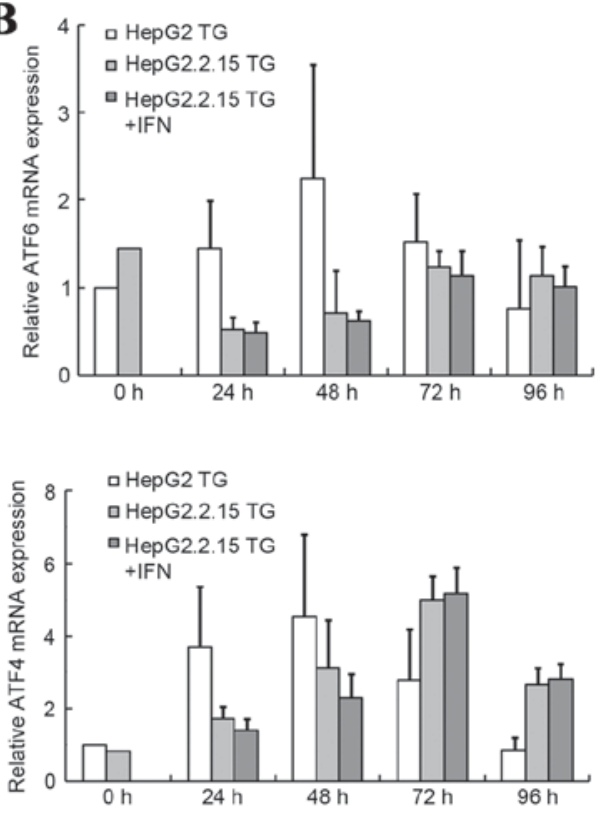

Figure 5. mRNA expression levels of genes in the 3 UPR pathways. (A) mRNA expression levels of CHOP and GADD34 were tested by qPCR, and XBP1 mRNA splicing was tested by reverse transcription PCR. (B) mRNA expression levels of ATF6 and ATF4 were tested by qPCR. Final abundances were adjusted to yield an arbitrary value of 1 for each gene in the HepG2 cells. mRNA, micro RNA; negative control; TG, thapsigargin; IFN, interferon; CHOP, DNA damage inducible transcript 3; GADD34, protein phosphatase 1 regulatory subunit 15A; XBP1, X-box binding protein; ATF4, activating transcription factor 4; ATF6, activating transcription factor 6; qPCR, quantitative polymerase chain reaction.
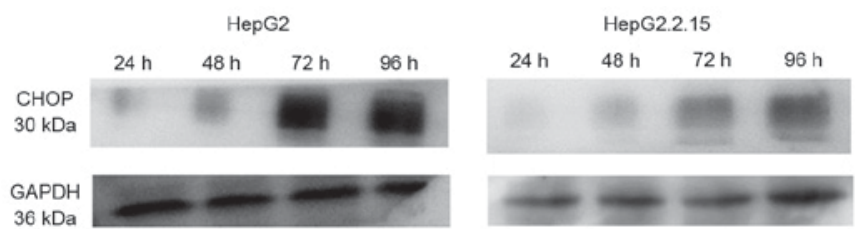

Figure 6. Western blot analysis of CHOP and GAPDH. Western blotting of CHOP, measuring $30 \mathrm{kDa}$, and GAPDH, measuring $36 \mathrm{kDa}$, in HepG2.2.15 and HepG2 cells subsequent to treatment with thapsigargin at each time point. GAPDH served as an internal control. CHOP, DNA damage inducible transcript 3; GADPH, glyceraldehyde 3-phosphate dehydrogenase.

XBP1 gene in the IRE1 pathway and the ATF4, CHOP and GADD34 genes in the PERK pathway, were examined. The ATF6, ATF4, CHOP and GADD34 genes were examined by qPCR, and XBP1 mRNA splicing was evaluated by RT-PCR. The results of these analyses are summarized in Fig. 5. The mRNA levels of CHOP, GADD34 and XBP1 increased significantly subsequent to TG treatment, as illustrated in Fig. 5A, and the mRNA levels of ATF6 and ATF4 exhibited increases, as demonstrated in Fig. 5B. According to this analysis, CHOP appeared to be the gene of greatest importance. Subsequent to TG treatment, the level of CHOP mRNA increased by 42 - to 95-fold in the HepG2 cells, and 10- to 30-fold in the HepG2.2.15 cells. At each time point, the level of CHOP mRNA expression was significantly higher in the HepG2 cells compared with the HepG2.2.15 cells $(\mathrm{P}<0.001)$. When the HepG2.2.15 cells were

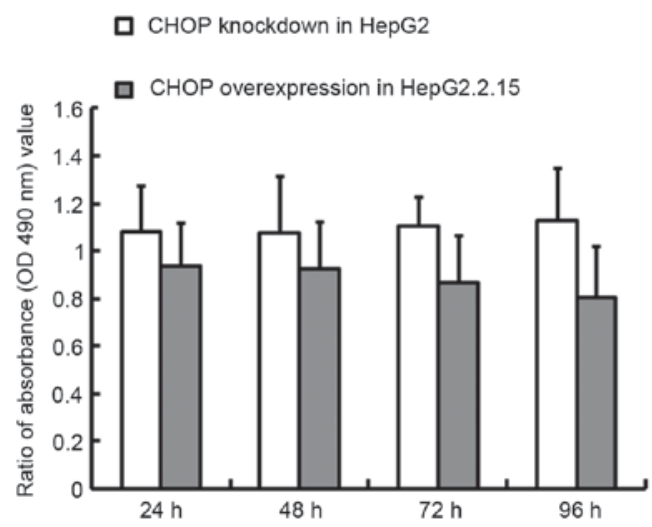

Figure 7. Cell proliferation measured by an MTT assay when CHOP was overexpressed in HepG2.2.15 cells or knocked down in HepG2 cells. Final abundances were calculated as the ratio of absorbance at $490 \mathrm{~nm}$ for cells overexpressing CHOP or with $\mathrm{CHOP}$ knockdown relative to cells treated with PBS at each time point. Data are presented as the mean \pm standard deviation from three independent experiments, and P-values were determined using an unpaired Student's t-test. OD, optical density; CHOP, DNA damage inducible transcript 3 .

treated with IFN $\alpha-2 \mathrm{~A}$ and TG, the CHOP mRNA expression levels were 1.16 and 1.11-fold higher than the expression levels without IFN $\alpha-2 \mathrm{~A}$ at 72 and $96 \mathrm{~h}(\mathrm{P}<0.05)$. Western blotting also confirmed that the protein levels of CHOP were higher in the HepG2 cells than in the HepG2.2.15 cells at each time point subsequent to treatment with TG, as demonstrated in Fig. 6. 
CHOP overexpression in HepG2.2.15 cells or knockdown in HepG2 cells affects proliferation. When CHOP was overexpressed in the HepG2.2.15 cells, the proliferation rate decreased by $6.5 \%$ at $24 \mathrm{~h}$ and $19.6 \%$ at $96 \mathrm{~h}$, relative to the wild-type cells $(\mathrm{P}<0.01)$, whilst in contrast, $\mathrm{CHOP}$ knockdown in the HepG2 cells increased proliferation $8.1 \%$ at $24 \mathrm{~h}$ and $12.5 \%$ at $96 \mathrm{~h}(\mathrm{P}<0.05)$, as demonstrated in Fig. 7.

\section{Discussion}

The results of the present study indicated that HBV may inhibit the apoptosis induced by ER stress via the repression of CHOP. CHOP is a member of the CCAAT/enhancer-binding protein (C/EBP) family of transcription factors (18), and functions as a dominant-negative inhibitor by forming heterodimers with other C/EBP members and preventing their DNA binding activity (19). CHOP is activated by ER stress, and promotes apoptosis. The activation of PERK increases the level of the phosphorylation of eukaryotic initiation factor 2 (eIF2), leading to an increase in the level of ATF4 translation. In turn, ATF4 induces the expression of CHOP (18). ATF4 and CHOP transactivate GADD34 (20), which selectively dephosphorylates eIF $2 \alpha$, completing a negative feedback loop and promoting the translation of other UPR genes. Whether CHOP promotes or inhibits oncogenesis is controversial. A previous study has revealed that pharmacological ER stresses that induce CHOP may kill cancer cells, including hepatomas, in vitro (21). However, in additional studies, CHOP appears to promote oncogenesis $(22,23)$. In the present study, it was demonstrated that increased levels of CHOP expression may have promoted HCC cell apoptosis, as summarized in Figs. 2-7, suggesting an antitumor role of CHOP.

According to a previous study (13), HBV induces ER stress independently, but the regulatory mechanisms of HBV-infected cells may be activated to reduce ER stress. Previous studies have investigated the pathological effect of HBV surface protein expression on the liver. In the livers of BALB/c transgenic mice, the expression of the HBV surface protein activates the PERK pathway and results in the expression of CHOP, leading to more extensive liver injury and fibrosis compared with transgenic mice with the C57BL/6 background (24). In another study using hepatoma cells, HBV small surface proteins triggered UPR, activated the PERK pathway and induced the phosphorylation of eIF $2 \alpha$, which promotes the expression of CHOP (25). In TG treated HepG2.2.15 cells, the present study demonstrated that HBV reduces the expression of $\mathrm{CHOP}$. This affects liver cancer cell apoptosis.

The present study contained a number of limitations. HepG2.2.15 cells were derived from HepG2 cells, and were stably transformed with 2 copies of the HBV genome (26). The culture medium of the HepG2.2.15 cells stably expressed HBV particles, hepatitis B surface antigen and Hepatitis B envelope antigen, but at low concentrations. Therefore, when the cells were treated with IFN $\alpha-2 \mathrm{~A}$, the antiviral effect was not measured due to the baseline HBV concentration being low. Additionally, these HBV markers are encoded by 2 copies of the HBV genome, stably transformed into the genomes, which is dissimilar to the natural progression of HBV infection in the human liver. Previously, the $\mathrm{Na}^{+}$-taurocholate cotransporting polypeptide (NTCP) was identified as a functional receptor for human HBV, a topic that requires attention (27). In future studies, HepG2 cells may be transfected with NTCP to increase the expression of HBV in the culture medium, simulating the natural history of HBV infection. In vivo studies should also be conducted to verify the role of HBV during ER stress.

In conclusion, the present study demonstrated that HBV may inhibit the cell apoptosis induced by ER stress, which is important for the development of ER stress based antitumor therapies for patients with HBV.

\section{Acknowledgements}

The present study was supported by grants from the National Basic Research Program of China (973 Program; grant nos., 2012CB519005 and 2013CB944903).

\section{References}

1. Toyoshima C, Nomura $\mathrm{H}$ and Sugita Y: Crystal structures of $\mathrm{Ca} 2+-\mathrm{ATPase}$ in various physiological states. Ann N Y Acad Sci 986: 1-8, 2003.

2. Winther AM, Liu H, Sonntag Y, Olesen C, le Maire M, Soehoel H, Olsen CE, Christensen SB, Nissen P and Møller JV: Critical roles of hydrophobicity and orientation of side chains for inactivation of sarcoplasmic reticulum Ca2+-ATPase with thapsigargin and thapsigargin analogs. J Biol Chem 285: 28883-28892, 2010.

3. Rajapaksa G, Nikolos F, Bado I, Clarke R, Gustafsson JÅ and Thomas C: ER $\beta$ decreases breast cancer cell survival by regulating the IRE1/XBP-1 pathway. Oncogene 34: 4130-4141, 2014.

4. Senft D and Ronai ZA: UPR, autophagy, and mitochondria crosstalk underlies the ER stress response. Trends Biochem Sci 40: 141-148, 2015.

5. Romero-Ramirez L, Cao H, Regalado MP, Kambham N, Siemann D, Kim JJ, Le QT and Koong AC: X box-binding protein 1 regulates angiogenesis in human pancreatic adenocarcinomas. Transl Oncol 2: 31-38, 2009.

6. Denmeade SR, Mhaka AM, Rosen DM, Brennen WN, Dalrymple S, Dach I, Olesen C, Gurel B, Demarzo AM, Wilding G, et al: Engineering a prostate-specific membrane antigen-activated tumor endothelial cell prodrug for cancer therapy. Sci Transl Med 4: 140ra186, 2012.

7. Park SW and Ozcan U: Potential for therapeutic manipulation of the UPR in disease. Semin Immunopathol 35: 351-373, 2013.

8. Chen CJ and Chen DS: Interaction of hepatitis B virus, chemical carcinogen, and genetic susceptibility: Multistage hepatocarcinogenesis with multifactorial etiology. Hepatology 36: 1046-1049, 2002.

9. Wang WA, Groenendyk J and Michalak M: Endoplasmic reticulum stress associated responses in cancer. Biochim Biophys Acta 1843: 2143-2149, 2014.

10. Liu YH, Weng YP, Lin HY, Tang SW, Chen CJ, Liang CJ, Ku CY and Lin JY: Aqueous extract of Polygonum bistorta modulates proteostasis by ROS-induced ER stress in human hepatoma cells. Sci Rep 7: 41437, 2017.

11. Tameire F, Verginadis II and Koumenis C: Cell intrinsic and extrinsic activators of the unfolded protein response in cancer: Mechanisms and targets for therapy. Semin Cancer Biol 33: 3-15, 2015.

12. El-Serag HB and Rudolph KL: Hepatocellular carcinoma: Epidemiology and molecular carcinogenesis. Gastroenterology 132: 2557-2576, 2007.

13. Lazar C, Uta M and Branza-Nichita N: Modulation of the unfolded protein response by the human hepatitis B virus. Front Microbiol 5: 433, 2014.

14. Sunami Y, Ringelhan M, Kokai E, Lu M, O'Connor T, Lorentzen A, Weber A, Rodewald AK, Mullhaupt B, Terracciano L, et al: Canonical NF- $\mathrm{B}$ signaling in hepatocytes acts as a tumor-suppressor in hepatitis B virus surface antigen-driven hepatocellular carcinoma by controlling the unfolded protein response. Hepatology 63: 1592-1607, 2016. 
15. Yeganeh B, Rezaei Moghadam A, Alizadeh J, Wiechec E Alavian SM, Hashemi M, Geramizadeh B, Samali A, Bagheri Lankarani K, Post M, et al: Hepatitis B and C virus-induced hepatitis: Apoptosis, autophagy, and unfolded protein response. World J Gastroenterol 21: 13225-13239, 2015

16. Livak KJ and Schmittgen TD: Analysis of relative gene expression data using real-time quantitative PCR and the 2(-Delta Delta C(T)) method. Methods 25: 402-408, 2001.

17. Lin JH, Li H, Yasumura D, Cohen HR, Zhang C, Panning B, Shokat KM, Lavail MM and Walter P: IRE1 signaling affects cell fate during the unfolded protein response. Science 318: 944-949, 2007.

18. Li Y, Guo Y, Tang J, Jiang J and Chen Z: New insights into the roles of CHOP-induced apoptosis in ER stress. Acta Biochim Biophys Sin (Shanghai) 46: 629-640, 2014.

19. Sheedy C, Mooney C, Jimenez-Mateos E, Sanz-Rodriguez A, Langa E, Mooney C and Engel T: De-repression of myelinregulating gene expression after status epilepticus in mice lacking the C/EBP homologous protein CHOP. Int J Physiol Pathophysiol Pharmacol 6: 185-198, 2014.

20. Marciniak SJ, Yun CY, Oyadomari S, Novoa I, Zhang Y, Jungreis R, Nagata K, Harding HP and Ron D: CHOP induces death by promoting protein synthesis and oxidation in the stressed endoplasmic reticulum. Genes Dev 18: 3066-3077, 2004.

21. Moon DO,Park SY, Choi YH, Ahn JS and Kim GY: Guggulsterone sensitizes hepatoma cells to TRAIL-induced apoptosis through the induction of CHOP-dependent DR5: Involvement of ROS-dependent ER-stress. Biochem Pharmacol 82: 1641-1650, 2011.
22. Crozat A, Aman P, Mandahl N and Ron D: Fusion of CHOP to a novel RNA-binding protein in human myxoid liposarcoma. Nature 363: 640-644, 1993.

23. Panagopoulos I, Höglund M, Mertens F, Mandahl N, Mitelman F and Aman P: Fusion of the EWS and CHOP genes in myxoid liposarcoma. Oncogene 12: 489-494, 1996.

24. Churin Y, Roderfeld M, Stiefel J, Würger T, Schröder D, Matono T, Mollenkopf HJ, Montalbano R, Pompaiah M, Reifenberg K, et al: Pathological impact of hepatitis B virus surface proteins on the liver is associated with the host genetic background. PLoS One 9: e90608, 2014.

25. Li J, Liu Y, Wang Z, Liu K, Wang Y, Liu J, Ding H and Yuan Z: Subversion of cellular autophagy machinery by hepatitis B virus for viral envelopment. J Virol 85: 6319-6333, 2011.

26. Sells MA, Chen ML and Acs G: Production of hepatitis B virus particles in Hep G2 cells transfected with cloned hepatitis B virus DNA. Proc Natl Acad Sci USA 84: 1005-1009, 1987.

27. Yan H, Zhong G, Xu G, He W, Jing Z, Gao Z, Huang Y, Qi Y, Peng B, Wang $\mathrm{H}$, et al: Sodium taurocholate cotransporting polypeptide is a functional receptor for human hepatitis B and D virus. Elife 1: e00049, 2012. 